\title{
Journal of Ophthalmic Surgery
}

ISSN: 2578-7160

Research Article

DOI: $10.36959 / 587 / 598$

\section{Outcomes of Entropion Repair in Ocular Cicatricial Pemphigoid Compared to Other Cicatricial Etiologies: A Retrospective Study}

\author{
Hayley A Braun, $M D, M P H^{1 \#}$, Lauren Schaffer, $M D^{2 \#}$, Emily F Cole, $M D, M P H^{1,3}$, Benjamin $B$ \\ Risk, $P h D^{4}$, Hee Joon Kim, $M D^{2}$ and Ron Feldman $M D, P h D^{1,4^{*}}$
}

${ }^{1}$ Department of Dermatology, Emory University School of Medicine, Atlanta, USA

${ }^{2}$ Department of Ophthalmology, Emory University School of Medicine, Atlanta, USA

${ }^{3}$ Regional Telehealth Service, VA Southeast Network VISN 7, Decatur, USA

${ }^{4}$ Department of Biostatistics and Bioinformatics, Rollins School of Public Health, Emory University, Atlanta, USA

${ }^{\#}$ Equal Contribution

\begin{abstract}
Background: Ocular cicatricial pemphigoid (OCP) can lead to severe conjunctival scarring and cicatricial entropion, which often necessitates repair. Surgical manipulation can increase the risk of inflammation, scarring, and disease progression. The objective of this study was to understand the characteristics of OCP patients who received entropion repairs and determine the difference in entropion recurrence or revision in patients with OCP or other cicatricial entropion repair.

Methods: A retrospective chart review was performed from January 2010 to June 2020. OCP patients from an autoimmune blistering disease clinic with entropion repair were compared to patients with repair of cicatricial entropion of other etiology. A generalized estimating equation assessed for evidence of recurrence of entropion or required revision.

Results: 19 OCP patients with cicatricial entropion and 60 patients with cicatricial entropion secondary to other etiologies received 38 and 75 entropion repairs, respectively. No difference in prevalence of revision or recurrence was found between groups.

Conclusion: This study provides evidence that in a population of OCP patients where the majority were systemically immunosuppressed, the likelihood of entropion recurrence or further repair was no different than that for repair of cicatricial entropion of other etiology.
\end{abstract}

Keywords

Ocular cicatricial pemphigoid, Cicatricial entropion, Mucous membrane pemphigoid

\section{Introduction}

Mucous membrane pemphigoid (MMP), a systemic autoimmune blistering disease, is characterized by recurrent blistering of the skin and mucous membranes [1]. When MMP affects the eye it is termed ocular cicatricial pemphigoid (OCP) [1]. OCP is a rare condition, with a reported incidence between 7 and 11 patients per 10,000 [2]. The ocular manifestations of OCP occur due to recurrent blister formation secondary to disruption of the adhesions between conjunctival epithelium and sub-epithelium, seen as linear deposits of immunoglobulins such as $\lg G, \lg A$, and IgM autoantibodies in the basement membrane zone on direct immunofluorescence [2,3]. The resultant inflammation of the conjunctiva typically presents as chronic inflammatory conjunctivitis that progresses to fibrosis and can potentially lead to blindness $[4,5]$.

*Corresponding author: Ron Feldman, MD, PhD, Department of Dermatology, Emory University School of Medicine, 1525 Clifton Road NE, $3^{\text {rd }}$ floor, Atlanta, GA 30322, USA, Tel: (404)778-3333

Accepted: October 29, 2021

Published online: October 31, 2021

Citation: Braun HA, Schaffer L, Cole EF, et al. (2021) Outcomes of Entropion Repair in Ocular Cicatricial Pemphigoid Compared to Other Cicatricial Etiologies: A Retrospective Study. J Ophthalmic Surg 4(1):43-47

Copyright: (C) 2021 Braun HA, et al. This is an open-access article distributed under the terms of the Creative Commons Attribution License, which permits unrestricted use, distribution, and reproduction in any medium, provided the original author and source are credited. 
Citation: Braun HA, Schaffer L, Cole EF, et al. (2021) Outcomes of Entropion Repair in Ocular Cicatricial Pemphigoid Compared to Other Cicatricial Etiologies: A Retrospective Study. J Ophthalmic Surg 4(1):43-47

Multiple eyelid abnormalities including trichiasis, lagophthalmos, symblepharon, and entropion can form as a result of the cicatrization, or scarring. These, in turn, can often further the progression of OCP by causing increased local irritation and inflammation that usually necessitates surgical repair [1,3]. However, perioperative control of ocular inflammation is essential, as it is thought that surgical trauma can result in increased inflammation, scarring, and disease progression [1].

Cicatricial entropion can also result from insults such as trauma, burns, and infection [6]. With cicatricial entropion secondary to non-autoimmune inflammatory causes, surgical procedures may be more successful due to lack of active inflammation [1]. One study assessed entropion repairs outcomes among 11 operated eyes across seven patients with OCP and found 1 (14\%) patient required a secondary repair on both eyes $(2 / 11$ ( $18 \%)$ of eyes operated on) [1]. However, the outcomes of surgical repair for OCP-related entropion repair compared to that for other cicatricial entropion has yet to be elucidated. This study aims to describe the characteristics of patients with OCP who received entropion repairs and determine the difference in entropion recurrence in patients with OCP versus patients with cicatricial entropion of other etiology.

\section{Methods}

After obtaining approval and a waiver of consent from the Institutional Review Board, a retrospective chart review was performed in the departments of ophthalmology and dermatology at the Emory University School of Medicine. OCP patients were identified as those with a diagnosis of OCP (including clinical and biopsy negative OCP) through the Autoimmune Blistering Disease Clinic in the dermatology department and cross-referenced with those that underwent an entropion repair at the Emory Eye Clinic between January 2010 and June 2020. OCP patients were excluded if they received a prior entropion repair at an outside hospital or if pertinent information was not available in their records. Patients that underwent repair with other causes of cicatricial entropion were identified by CPT code 67924 or 67825 between January 2010 and June 2020. Exclusion criteria for the non-OCP group included age less than 18-years-old at the time of repair, biopsy-negative OCP, including all patients receiving conjunctival or eyelid direct immunofluorescence (DIF), senile or suspected senile entropion repair, previous entropion repair at an outside hospital or at this institution prior to 2010, and if no postoperative follow-up occurred.

Demographic data including sex and age was collected from electronic medical records. Race was not included due to high percentage of missing in the non-OCP patients (23\%). OCP-related data included conjunctival biopsy immunofluorescence, indirect immunofluorescence, ELISA, other clinical sites of disease involvement, systemic immunosuppressive medication at time of surgery, ophthalmologic medications at time of surgery, and any history of rituximab. If multiple immunofluorescence results were reported, all relevant positives were reported. Ophthalmic medication history was recorded from the ophthalmology visit immediately prior to surgery. Ophthalmic exam findings were collected from the oculoplastics pre-operative visit and at follow-up visits 2 and 3 post-surgery (usually reflecting 1- and 3-month follow-ups). The first follow-up exams at 1 week were excluded as these were cursory wound checks. Subsequent visits were analyzed for any evidence of entropion recurrence. Surgical repairs were classified into the following categories: a rotational procedure, a Wies procedure (a transverse blepharotomy with marginal rotation), a grafting procedure (i.e. mucous membrane, amniotic membrane, ear cartilage, etc.), or other. If a Wies procedure also included a graft, it was categorized as a Wies procedure.

\section{Statistical analysis}

Chi-square tests were run to assess demographic differences between groups. For tests with cell size $<5$, Fisher's exact was used. Mean age was calculated and nonparametric Wilcoxon t-test estimation (two-sided) were run. Statistical analysis of differences in ophthalmologic findings between groups was not performed due to the sparsity of data.

A generalized estimating equation with binomial likelihood and logit link was used to analyze the association between a diagnosis of OCP and failure of the first entropion repair. Failure of first repair was measured as either recurrence or repair noted. Patient ID was included as the clustering variable with exchangeable correlation structure. We fit an unadjusted model with OCP diagnosis only and an adjusted model including age at first repair, sex, repair approach as described in surgical note, systemic steroid use, non-steroidal systemic immunosuppressants, ophthalmologic steroids, and non-steroidal ophthalmologic immunosuppressants. Collinearity between potential confounders was assessed by correlation $>0.7$. Systemic steroids and systemic nonsteroidal immunosuppressive agents were highly correlated with a diagnosis of OCP and were removed from the model. Statistical analysis was performed using SAS 9.4 (Cary, NC) and geepack in R 4.0.3 [7,8].

\section{Results}

19 patients with OCP and 60 patients without OCP met inclusion criteria and received a first-time entropion repair at this institution. Immune characteristics of OCP patients are displayed in Supplementary Table 1. Of the 15 who had an ocular direct immunofluorescence, 14 (93\%) had evidence of immune deposition.

The 19 OCP patients underwent 38 separate eyelid repairs (Supplementary Table 2). Systemic immunosuppression (steroids, methotrexate (MTX), mycophenolate mofetil(MMF), cyclosporine, azathioprine (AZA), or cyclophosphamide) was documented prior to surgery in 35 (92\%) of lid repairs (representing 17 (89\%) of patients). The most commonly used non-steroidal immunosuppressant was MMF 29 (76\%) of lid repairs, representing 13 (68\%) of patients).

Patient populations did not differ by sex (Table 1). Age at first repair differed significantly by group while the eyelid repaired and procedure type did not differ by group. The 
Citation: Braun HA, Schaffer L, Cole EF, et al. (2021) Outcomes of Entropion Repair in Ocular Cicatricial Pemphigoid Compared to Other Cicatricial Etiologies: A Retrospective Study. J Ophthalmic Surg 4(1):43-47

Table 1: Demographic comparison of OCP-related entropion versus other cicatricial entropion repair patients

\begin{tabular}{|c|c|c|c|}
\hline & OCP & $\begin{array}{l}\text { Cicatricial Entropion of Other } \\
\text { Etiology }\end{array}$ & \\
\hline Patient Demographics & $\mathrm{N}$ persons $=19$ & $\mathrm{~N}$ persons $=60$ & P-value \\
\hline \multicolumn{4}{|l|}{ Gender } \\
\hline Male & $8(42.1 \%)$ & 32 (53.3\%) & 0.39 \\
\hline Female & $11(57.9 \%)$ & $28(46.7 \%)$ & \\
\hline Surgery Specific Characteristics & $\mathrm{N}$ eyelids $=38$ & N eyelids $=75$ & \\
\hline \multicolumn{4}{|l|}{ Eyelid } \\
\hline RUL & $10(26.3 \%)$ & $14(18.7 \%)$ & 0.42 \\
\hline RLL & $11(29 \%)$ & $16(21.3 \%)$ & \\
\hline LUL & $7(18.4 \%)$ & $23(30.7 \%)$ & \\
\hline LLL & $10(26.3 \%)$ & $22(29.3 \%)$ & \\
\hline \multicolumn{4}{|l|}{ Age at time of surgery (years) $\mathrm{N}(\%)$} \\
\hline Mean (SD) & $66.1(9.2)$ & $56.8(19.3)$ & \\
\hline$<55$ & $4(10.5 \%)$ & $31(41.3 \%)$ & 0.001 \\
\hline $55-64$ & $16(42.1 \%)$ & $11(14.7 \%)$ & \\
\hline $65-74$ & $11(29 \%)$ & $23(30.7 \%)$ & \\
\hline$\geq 75$ & $7(18.4 \%)$ & $10(13.3 \%)$ & \\
\hline \multicolumn{4}{|l|}{ Procedure Type } \\
\hline Wies $^{*}$ & $23(60.5 \%)$ & $48(64 \%)$ & $0.07^{* *}$ \\
\hline Graft (amniotic, mucous, ear cartilage, etc) & $4(10.5 \%)$ & $17(22.7 \%)$ & \\
\hline Other & $11(29 \%)$ & $10(13.3 \%)$ & \\
\hline
\end{tabular}

*Wies is a transverse blepharotomy with marginal rotation. These include modified Wies with partial thickness blepharotomy and Wies with mucous membrane graft.

${ }^{* *}$ Fisher's exact used if any cell size $<5$

Table 2: Ophthalmologic outcomes of OCP-related entropion versus other cicatricial entropion repair patients

\begin{tabular}{|c|c|c|c|c|c|c|}
\hline & \multicolumn{2}{|c|}{ Pre-Op } & \multicolumn{2}{|c|}{ Post-Op 2} & \multicolumn{2}{|c|}{ Post-Op 3} \\
\hline & OCP & $\begin{array}{l}\text { Other } \\
\text { Cicatricial }\end{array}$ & OCP & $\begin{array}{l}\text { Other } \\
\text { Cicatricial }\end{array}$ & OCP & $\begin{array}{l}\text { Other } \\
\text { Cicatricial }\end{array}$ \\
\hline Avg time pre/post surgery (weeks) & $\begin{array}{l}8.3(5.2) \\
N=38\end{array}$ & $\begin{array}{l}7.6(4.4) \\
N=75\end{array}$ & $\begin{array}{l}7.4(7.5) \\
N=34\end{array}$ & $\begin{array}{l}6.9(3.2) \\
N=62\end{array}$ & $\begin{array}{l}21.5(12.6) \\
\mathrm{N}=21\end{array}$ & $\begin{array}{l}17.1(11.5) \\
N=24\end{array}$ \\
\hline \multicolumn{7}{|l|}{ Ophthalmic Exam } \\
\hline Visual Acuity Logmar (mean (SD)) & $\begin{array}{l}0.35(0.37) \\
N=34\end{array}$ & $\begin{array}{l}0.29(0.30) \\
N=44\end{array}$ & $\begin{array}{l}0.34(0.35) \\
N=29\end{array}$ & $\begin{array}{l}0.40(0.50) \\
N=33\end{array}$ & $\begin{array}{l}0.22(0.15) \\
N=16\end{array}$ & $\begin{array}{l}0.21(0.13) \\
N=12\end{array}$ \\
\hline Abnormal Motility Exam & 1 out of 17 & 8 out of 32 & 0 out of 1 & 1 out of 7 & 0 out of 6 & 0 out of 5 \\
\hline External N (\%) & $N=35$ & $N=73$ & $\mathbf{N}=\mathbf{3 0}$ & $N=53$ & $N=19$ & $N=18$ \\
\hline Entropion & 34 (97.14\%) & $(100.00 \%)$ & $3(10.00 \%)$ & $2(3.77 \%)$ & $4(21.05 \%)$ & $2(11.11 \%)$ \\
\hline Fornix Shortening & $5(14.29 \%)$ & $3(4.11 \%)$ & $1(3.33 \%)$ & $1(1.89 \%)$ & $0(0 \%)$ & $0(0 \%)$ \\
\hline Ptosis & $5(14.29 \%)$ & $4(5.48 \%)$ & $0(0 \%)$ & $1(1.89 \%)$ & $0(0 \%)$ & $2(11.11 \%)$ \\
\hline Lid Margin N (\%) & $\mathbf{N}=\mathbf{2 7}$ & $N=54$ & $N=25$ & $N=40$ & $\mathbf{N}=\mathbf{2 0}$ & $N=18$ \\
\hline Trichiasis & $11(40.74 \%)$ & $31(57.41 \%)$ & $2(8.00 \%)$ & $5(12.50 \%)$ & $6(30.00 \%)$ & $0(0 \%)$ \\
\hline Distichiasis & $5(18.52 \%)$ & $2(3.70 \%)$ & $2(8.00 \%)$ & $0(0 \%)$ & $2(10.00 \%)$ & $1(5.55 \%$ \\
\hline Conjunctiva Exam Findings & $N=29$ & $N=49$ & $N=22$ & $N=33$ & $N=19$ & $N=15$ \\
\hline Injection & 7 (24.14\%) & $13(26.53 \%)$ & $0(0 \%)$ & $5(15.15 \%)$ & $2(10.53 \%)$ & $2(13.33 \%)$ \\
\hline
\end{tabular}


Citation: Braun HA, Schaffer L, Cole EF, et al. (2021) Outcomes of Entropion Repair in Ocular Cicatricial Pemphigoid Compared to Other Cicatricial Etiologies: A Retrospective Study. J Ophthalmic Surg 4(1):43-47

\begin{tabular}{|l|l|l|l|l|l|l|}
\hline Symblepharon & $13(44.83 \%)$ & $12(24.49 \%)$ & $7(31.82 \%)$ & $2(6.06 \%)$ & $5(26.31 \%)$ & $1(6.67 \%)$ \\
\hline Slit-lamp N (\%) & $\mathbf{N = 1 0}$ & $\mathbf{N = 2 8}$ & $\mathbf{N = 7}$ & $\mathbf{N = 1 8}$ & $\mathbf{N = 5}$ & $\mathbf{N = 5}$ \\
\hline Punctate Epithelial Erosions (PEE) & $5(50 \%)$ & $14(50.00 \%)$ & $2(28.57 \%)$ & $3(16.67 \%)$ & $2(40.00 \%)$ & $4(80.00 \%)$ \\
\hline Punctate Epithelial Keratophathy (PEK) & $3(30 \%)$ & $4(14.29 \%)$ & $1(14.29 \%)$ & $2(11.11 \%)$ & $0(0 \%)$ & $0(0 \%)$ \\
\hline Superficial Punctate Keratitis (SPK) & $2(20 \%)$ & $1(3.57 \%)$ & $0(0 \%)$ & $0(0 \%)$ & $0(0 \%)$ & $0(0 \%)$ \\
\hline Neovascularization & $1(10 \%)$ & $2(7.14 \%)$ & $1(14.29 \%)$ & $8(44.44 \%)$ & $1(20.00 \%)$ & $2(40.00 \%)$ \\
\hline Epithelial defect and/or ulcer & $0(0 \%)$ & $1(3.57 \%)$ & $3(42.86 \%)$ & $1(5.56 \%)$ & $1(20.00 \%)$ & $0(0 \%)$ \\
\hline
\end{tabular}

most commonly performed procedure among both groups was a Wies procedure.

Ophthalmic exam findings for the eyelid undergoing repair were collected from the oculoplastics clinic notes. However, documentation of examinations were inconsistent in their inclusion of ocular vitals, external exam findings, lid margin exam, exam of the conjunctiva, and slit-lamp findings (Table 2). At the pre-operative appointment, mean best-corrected visual acuity was 0.35 and $0.29 \log$ MAR in the OCP cohort and other cicatricial entropion cohort, respectively. After lid surgery, the average best-corrected visual acuity in the operated eyes for both cohorts was $0.21 \log$ MAR. A common pre-operative examination finding was punctate epithelial erosions (PEE), which was found in $50 \%$ of patients in both groups with a documented slit-lamp exam. Symblepharon was noted on pre-operative exam in $44.83 \%$ of the OCP group with a conjunctival exam documented, compared to just $24.49 \%$ of the eyelids with cicatricial entropion of a different etiology. Additionally, trichiasis was present on pre-operative examination in $40.74 \%$ of the OCP cohort and $57.41 \%$ of the comparison group with documented lid margin exams. Of note, trichiasis was noted post-operatively in $30 \%$ of OCP patients and $0 \%$ in the non-OCP group.

The unadjusted odds of recurrence or revision was higher in patients with OCP diagnosis than in those with no OCP diagnosis, but this result was not significant (unadjusted odds ratio (OR) 2.66, 95\% confidence interval $(\mathrm{Cl})$ 0.92-7.69, $\mathrm{p}=$ 0.07 ). There was also no significant effect of OCP diagnosis when controlling for age, sex, ophthalmologic steroids, nonsteroidal ophthalmic immunosuppression, and entropion repair approach (adjusted OR: 2.43, 95\% Cl: 0.75-7.87). Age at first repair was positively associated with revision or recurrence $(p=0.026)$. The use of ophthalmic steroids decreased the odds of recurrence or revision (OR: $0.2795 \%$ $\mathrm{Cl}$ : 0.07-0.96, $\mathrm{p}=0.045)$. Mean follow-up time based on last oculoplastics notes was 83 weeks (SD $=67$ ) and 50 weeks (SD $=70$ ) for OCP and non-OCP patients, respectively.

\section{Discussion}

Ocular cicatricial pemphigoid is a chronic autoimmune inflammatory condition causing recurrent blistering and inflammation with subsequent scarring of the ocular surface that can potentially lead to blindness [1]. Among the many ophthalmic manifestations found in $\mathrm{OCP}$, cicatricial entropion can form as the posterior lamella of the eyelid scars and contracts causing the eyelid to turn inward [9]. The entropion ought to be repaired in order to prevent further corneal damage from the continuous ocular surface irritation, as well as to improve patient comfort. It is generally accepted in OCP patients that surgery should ideally be attempted when the inflammation is controlled, in order to prevent potential disease exacerbation triggered by post-surgical inflammation $[1,10,11]$. However, to our knowledge the success rate of surgical repair of cicatricial entropion secondary to OCP, whether adequately controlled with systemic medication or not, has not been previously compared to the results of repair of cicatricial entropion secondary to other etiologies. Thus, this study aimed to examine the results of entropion repair in the two populations, and to determine whether patients with cicatricial entropion secondary to OCP had a recurrence or required a revision more frequently than those with cicatricial entropion secondary to another etiology.

The results show that the likelihood of recurrence or revision did not differ between the OCP-related entropion and entropion of other etiology. This was unexpected as the authors theorized that the underlying inflammatory response in OCP-related repair would increase the likelihood of required revision or recurrence. One explanation for this finding is that almost all patients in this study with OCP were on immunosuppressive medications, thus preventing an exaggerated immune response to the surgical repair. Due to the high collinearity between the use of systemic (both steroidal and non-steroidal) immunosuppression within the OCP patients, the separate effects of these medications cannot be statistically assessed. The use of ophthalmic steroids may be associated with lower likelihood of revision or recurrence.

These findings would suggest that in a population of OCP patients on immunosuppressive medications, entropion repair outcomes are similar to those receiving repair for other etiologies. This aligns with previous findings of Gibbons, et al. comparing entropion repairs among 11 operated eyes across seven patients with $\mathrm{OCP}$, which found only one patient required repair during follow-up [1]. All seven patients were on either cyclophosphamide or mycophenolate mofetil (MMF), 4 with concomitant prednisone use. These findings support the previous recommendation that OCP patients should receive systemic immunosuppressive therapy when undergoing entropion repair [1]. Furthermore, it is worth noting that the majority of entropion repairs performed in both the OCP and comparison cohorts utilized a Wies procedure. This is significant because it has been previously suggested that entropion repairs in OCP patients should spare the conjunctiva in order to reduce the risk of inflammation and disease exacerbation [9]. Our results suggest that with adequate systemic disease control, the even a technique like 
Citation: Braun HA, Schaffer L, Cole EF, et al. (2021) Outcomes of Entropion Repair in Ocular Cicatricial Pemphigoid Compared to Other Cicatricial Etiologies: A Retrospective Study. J Ophthalmic Surg 4(1):43-47

a Wies could be successful. However, interpretation is limited due to lack of comparison to OCP patients without systemic immunosuppression.

Our data also shows post-operative trichiasis in OCP patients more so than our comparison cohort. One possible explanation for this increased recurrence of trichiasis in the OCP population is disease continuance. Trichiasis is one of the most common eyelid abnormalities in OCP and may occur independently of entropion [1]. The non-OCP cohort typically had entropion due to trauma, previous surgeries, or other single inciting events like Stevens-Johnsons Syndrome rather than from an underlying chronic condition, it is reasonable that the trichiasis did not continue to be seen on follow-up examinations. Another possible explanation is that OCP patients have a higher incidence of distichiasis, a clinically distinct finding where lashes grow out from the meibomian gland. Unlike trichiasis, distichiasis is not corrected by rotational lid procedures. The Emory EMR does not distinguish between distichiasis and trichiasis unless distichiasis is manually entered and thus distichiasis may be misclassified as trichiasis.

Strengths of this study include the description of a large OCP population undergoing entropion repair, all of whom follow in an autoimmune blistering disease clinic and many of whom have long-term follow-up. Limitations of this study include that its retrospective nature and thus the reliance on prior documentation in patients' medical records. Specifically, which components of the ophthalmic exam were included in each clinic note was inconsistent both between patients and between clinic visits for each patient. This inconsistency resulted in the inability to assess ophthalmologic findings pre- and post-repair for patient. However, due to the rarity of the disease, a prospective study would be very difficult to perform. Another limitation of this study is that 35 of the 38 entropion repairs in OCP patients included in this study had documentation of systemic immunosuppressive therapy prior to surgery. Thus, comparison of surgical outcomes between OCP patients with and without a history of immunosuppressive therapy is limited. Future prospective studies should assess complete ophthalmologic findings preand post-surgical repair to assess for other potential negative outcomes secondary to surgical entropion repair.

OCP can result in severe and destructive ocular complications such as entropion. One of the biggest concerns with surgical repair is the risk of triggering an even more severe inflammation, scarring, disease progression. This study provides evidence that entropion recurrence in a population of OCP patients where the majority were systemically immunosuppressed, the likelihood of recurrence or repair was no different than those due to other cicatrizing etiologies.

\section{Potential Conflicts of Interest}

Dr. Feldman has consulted for Astrazeneca and Alkahest and receives research support from Pfizer. Funders have no involvement in the study design; collection, analysis and interpretation of data; the writing of the manuscript; or the decision to submit the manuscript for publication. Dr. Braun, Dr. Schaffer, Dr. Cole, Dr. Risk, and Dr. Kim have no conflicts of interest to disclose.

\section{References}

1. Gibbons A, Johnson TE, Wester ST, et al. (2015) Management of patients with confirmed and presumed mucous membrane pemphigoid undergoing entropion repair. Am J Ophthalmol 159: 846-52.e2.

2. Morel M, DeGrazia T, Ward L, et al. (2020) Single Center Retrospective Study of Patients with Ocular Mucous Membrane Pemphigoid (MMP). Ocul Immunol Inflamm: 1-6.

3. Taurone S, Spoletini M, Ralli M, et al. (2019) Ocular mucous membrane pemphigoid: A review. Immunol Res 67: 280-289.

4. Fremont F, Pelissier-Suarez C, Fournié $P$, et al. (2019) Clinical Characteristics and Outcomes of Ocular Cicatricial Pemphigoid: A Cohort Study and Literature Review. Cornea 38: 1406-1411.

5. Wang K, Seitzman G, Gonzales JA (2018) Ocular cicatricial pemphigoid. Curr Opin Ophthalmol 29: 543-551.

6. Bergstrom R, Czyz CN (2021) Entropion. StatPearls. Treasure Island (FL): StatPearls.

7. Højsgaard S, Halekoh U, Yan J (2005) The R Package geepack for Generalized Estimating Equations 15: 11.

8. (2020) R: A Language and Environment for Statistical Computing [program]. Vienna, Austria: $\mathrm{R}$ Foundation for Statisical Computing, 2020.

9. Choung H, Reshef ER, Tanking T, et al. (2020) A conjunctivalsparing surgical technique for lower eyelid cicatricial entropion repair in ocular cicatricial pemphigoid. Orbit 39: 23-30.

10. Kirzhner M, Jakobiec FA (2011) Ocular cicatricial pemphigoid: A review of clinical features, immunopathology, differential diagnosis, and current management. Semin Ophthalmol 26: 270277.

11. Saw VP, Dart JK (2008) Ocular mucous membrane pemphigoid: Diagnosis and management strategies. Ocul Surf 6: 128-142.

DOI: $10.36959 / 587 / 598$

Copyright: (C) 2021 Braun HA, et al. This is an open-access article distributed under the terms of the Creative Commons Attribution License, which permits unrestricted use, distribution, and reproduction in any medium, provided the original author and source are credited. 